\title{
Education Of Females And Impact On Fertility
}

\author{
Rabiya Ali, Rehana Rehman
}

Dear Editor,

Infertility; an inability to conceive after unprotected intercourse for a period of at least one year, is associated with several medical conditions in both men and women which may end up in depression and a number of medical, psycho- social disorders ${ }^{1}$. It has been observed that female partners are usually blamed for this condition, especially when they are living in joint families ${ }^{2}$. Since we are aware of the fact that females in many settings are put into early marriage for the sake of royalty or for carrying forward the genetic legacy which prevents them from seeking higher or sometimes even basic education and thus ending up believing in misconceptions and myths. This renders them with lack of awareness and therefore a positive approach for diagnosis and management of infertility.

There is an increased number of studies done on Fertility and its relation to ethnicity and education but this are not much is done in Pakistan since the late $90{ }^{\prime} \mathrm{s}^{3}$. Infertility is not just related to illiteracy or less education but many welleducated women also suffer from infertility. As they focus on pursuing higher education and becoming career oriented hence delay parenthood, resulting in detoriation in natural child bearing process with increase in their age ${ }^{4}$. While achieving their goals they refrain themselves from the fundamental knowledge related to their own mental and physical health. On later realization, they face depression and anxiety and their apprehension adds on to the causes of infertility.

We conducted a survey to compare the level of education in fertile and infertile female population and observed that $13 \%$ infertile females were uneducated as compared to $4 \%$ fertile females. The importance of level of education with awareness to opt for fertility treatments is described in a study done in Toronto in $2014^{5}$. According to our results $51 \%$ fertile females were graduates as compared to $33 \%$ infertile females, which raises the question to explore the impact of education on awareness of fertility options and treatment plans.

We realize that targeted educational interventions to expand knowledge about the ideal age of fertility, different elements affecting fertility potential and fertility options can only be dispersed once the female is literate. We therefore advocate on emphasis of provision of basic and essential education to females from rural as well as urban population followed by motivational research programs to encourage women of all ethnic backgrounds to acquire education.

\section{REFERENCES:}

1. Nargund GJF, views, ObGyn vi. Why fertility education is needed in schools. 2015;7(3):189.

2. Alam F, Khan TA, Rehman R. Stress of infertility: Can the couple cope? JPMA The Journal of the Pakistan Medical Association 2018;68(4):679-80.

3. Sathar ZA. Does female education affect fertility behaviour in Pakistan? The Pakistan Development Review 1984:57390.

4. Mahey R, Gupta M, Kandpal S, Malhotra N, Vanamail P, Singh N, et al. Fertility awareness and knowledge among Indian women attending an infertility clinic: a cross-sectional study. BMC women's health 2018;18(1): 177.

5. Swift BE, Liu KE. The effect of age, ethnicity, and level of education on fertility awareness and duration of infertility. Journal of Obstetrics and Gynaecology Canada 2014;36(11):990-96. 